\title{
Resveratrol affects differently rat liver and brain mitochondrial bioenergetics and oxidative stress in vitro: Investigation of the role of gender
}

\author{
Ana C. Moreira ${ }^{\text {a,b,c }}$, Ana M. Silva ${ }^{\text {b,c }}$, Maria S. Santos ${ }^{\text {b,c }}$, Vilma A. Sardão ${ }^{\text {b,* }}$ \\ ${ }^{a}$ Doctoral Programme in Experimental Biology and Biomedicine, Center for Neuroscience and Cell Biology, University of Coimbra, Portugal \\ ${ }^{\mathrm{b}} \mathrm{CNC}$ - Center for Neuroscience and Cell Biology, University of Coimbra, Portugal \\ ${ }^{\mathrm{c}}$ Department of Life Sciences, University of Coimbra, Portugal
}

\section{A R T I C L E I N F O}

\section{Article history:}

Received 27 September 2012

Accepted 17 November 2012

Available online 29 November 2012

\section{Keywords:}

Resveratrol

Mitochondria

Brain

Liver

Toxicology

Gender

\begin{abstract}
A B S T R A C T
Resveratrol (3,5,4'-trihydroxy-trans stilbene) is commonly recognized by its antioxidant properties. Despite its beneficial qualities, the toxic effects of this natural compound are still unknown. Since mitochondria are essential to support the energy-dependent regulation of several cell functions, the objective of this study was to evaluate resveratrol effects on rat brain and liver mitochondrial fractions from male and females regarding oxidative stress and bioenergetics. No basal differences were observed between mitochondrial fractions from males and females, except in liver mitochondria, the generation of $\mathrm{H}_{2} \mathrm{O}_{2}$ by the respiratory chain is lower for female preparations. Resveratrol inhibited lipid peroxidation in preparations from both genders and organs. Furthermore, brain mitochondria in both gender groups appeared susceptible to resveratrol as seen by a decrease in state 3 respiration and alterations in mitochondrial membrane potential fluctuations during ADP phosphorylation. As opposed, liver mitochondria were less affected by resveratrol. Our data also demonstrates that resveratrol inhibits complex I activity in all mitochondrial preparations. The results suggest that brain mitochondria appear to be more susceptible to resveratrol effects, and gender appears to play a minor role. It remains to be determined if resveratrol effects on brain mitochondria contribute to deterioration of mitochondrial function or instead to mediate hormesis-mediated events.
\end{abstract}

(c) 2012 Elsevier Ltd. All rights reserved.

\section{Introduction}

The redox active polyphenol compound resveratrol (3,5,4'-trihydroxy-trans stilbene) was firstly identified in roots from white hellebore (Veratrum album) and later in roots from Japanese knotweed (Polygonum cuspidatum) (Nonomura et al., 1963). Resveratrol is also found in grapes (Vitis vinifera), grape juice, wine berries (Vaccinium macropon) and peanuts (Arachis hypogaea) (Fremont, 2000; Pervaiz, 2003). Resveratrol has been shown to trigger several physiological effects in laboratory animals, resulting in cancer prevention, microvascular and neuroprotection as well as antidiabetic

\footnotetext{
Abbreviations: ADP, adenosine diphosphate; ANT, adenosine nucleotide translocator; ATP, adenosine triphosphate; BBB, blood-brain barrier; DCPIP, 2,6dichorophenolindophenol; DMSO, dimethyl sulfoxide; DOX, doxorubicin; EDTA, ethylenediaminetetraacetic acid; EGTA, ethyleneglycoltetraacetic acid; FOXO1, forkhead box protein 1; LDL, low density protein; LPS, lipopolysaccharide; NADH, nicotinamide adenine dinucleotide; Ox, oxidized; RCR, respiratory control ratio; ROT, rotenone; SOD, superoxide dismutase; TBARS, thiobarbituric acid reactive species; $\mathrm{TPP}^{+}$, tetraphenylphosphonium.

* Corresponding author. Address: Center for Neuroscience and Cell Biology, Largo Marquês de Pombal, 3004-517 Coimbra, Portugal. Tel.: +351 239855 760; fax: +351 239853409.

E-mail address: vimarisa@ci.uc.pt (V.A. Sardão).
}

effects (Baur and Sinclair, 2006). Resveratrol is one of the main components of red wine, the consumption of which is associated with a lower incidence of heart failure in France (Richard, 1987). The protective effects are associated with antioxidant proprieties that were confirmed in the heart in different models (Gresele et al., 2011), including lipopolysaccharide (LPS)-induced oxidative stress (Sebai et al., 2011) and doxorubicin (DOX)-induced cardiotoxicity (Xu et al., 2012).

Resveratrol crosses the blood-brain barrier (BBB) (Wang et al., 2002), demonstrating neuroprotective effects in several disorders such as cerebral ischemia and Alzheimer's disease (Baur and Sinclair, 2006). Resveratrol also increases spatial memory performances in the circular platform tasks in primates (Dal-Pan et al., 2011), thus demonstrating cognitive and neuroprotective effects (Agarwal and Baur, 2011; Huber and Superti-Furga, 2011). Fukiu et al. showed that resveratrol induces the expression of mitochondrial superoxide dismutase (SOD2) and as a consequence reduces mitochondrial oxidative stress and damage in neurons (Fukui et al., 2010).

Specifically in the liver, resveratrol up-regulates the expression of glucogenic genes by attenuating insulin signaling and by deacetylating FOXO1 (Park et al., 2010). Resveratrol also decreases 
fibrosis and promotes hepatocyte regeneration, which increased the survival of mice during cholestatic liver injury (Chan et al., 2011). Although the protective effects of resveratrol on heart mitochondria have been described (Gutierrez-Perez et al., 2011), mitochondrial-specific studies are lacking in liver and brain mitochondria.

Mitochondria are the cell powerhouses due to the synthesis of ATP by oxidative phosphorylation (Pereira et al., 2009a). Mitochondria are also a major endogenous source of reactive oxygen species, either under normal (Rigoulet et al., 2011) or pathological (Puente-Maestu et al., 2012; Reale et al., 2012) conditions. Macromolecular oxidative damage in mitochondria induces a decline in the efficiency of oxidative phosphorylation, and result in the induction of the mitochondrial permeability transition and in the release of pro-apototic factors that trigger apoptosis (Pereira et al., 2009a). With this in mind, several effects of resveratrol on cells may be derived from direct or indirect mitochondrial effects. To support the evidence that resveratrol presents direct effects on mitochondria, we have isolated fractions from rat liver and brain and investigated whether resveratrol alters mitochondrial bioenergetics and prevents induced oxidative damage. A second important question was whether resveratrol-induced mitochondrial effects are gender-dependent. To answer this latter question, mitochondrial fractions were isolated from female and male rats. Isolated mitochondrial fractions are a recognized model to measure compound toxicity (Pereira et al., 2009b). Nevertheless, the large majority of experiments is performed with mitochondrial fractions from male animals. Differences between mitochondrial fractions from male and female animal models may influence the final outcome of chemical-biological interactions at the mitochondrial level. The concentrations used in this study are within the concentration range used by others (Annabi et al., 2012; He et al., 2012; Price et al., 2012; Valdecantos et al., 2010).

\section{Material and methods}

\subsection{Chemicals}

All chemicals used in this work were purchased from Sigma Aldrich Co (St. Louis, MO), unless specified. Resveratrol was prepared in DMSO, the final volume used was lower than $0.1 \%(\mathrm{v} / \mathrm{v})$; aqueous solutions were prepared in ultrapure water (Milli-Q Biocel A10 with pre-treatment via Elix 5, Millipore, Billerica, MA, USA). Non-aqueous solutions were prepared in ethanol. In this case, the final volume used was always lower than $0.1 \%(\mathrm{v} / \mathrm{v})$.

\subsection{Animals}

Male and female Wistar rats (8-12 weeks) from our animal colony (Center for Neuroscience and Cell Biology, University of Coimbra) were housed in type III-H cages (Tecniplast, Italy) with irradiated corn cob grit bedding (Scobis Due, Mucedola, Italy), following environmental requirements with ad libitum acess to food (4RF21, Mucedola, Italy) and water and maintained at constant temperature $\left(22^{\circ} \mathrm{C}\right)$ and humidity with a $12 \mathrm{~h}$ light/dark cycle. Animal handling and sacrifice followed the procedures approved by the Federation of European Laboratory Animal Science Associations (FELASA). Animal handlers and the authors ACM and VAS are credited by FELASA (category $C$ ) for animal experimentation.

\subsection{Mitochondria preparation}

The livers were quickly removed and mitochondria were isolated by conventional methods (Moreno et al., 2007). Briefly, liver mitochondria were isolated using an homogenization media composed of $250 \mathrm{mM}$ sucrose, $5 \mathrm{mM}$ Hepes, $0.5 \mathrm{mM}$ EGTA and $0.1 \%$ defatted bovine serum albumin containing medium $(\mathrm{pH}=7.2)$. The mitochondrial pellet was washed twice and suspended in washing buffer (250 mM sucrose, $10 \mathrm{mM}$ HEPES, $\mathrm{pH}=7.4$ ). Brain mitochondria were isolated by a previously published method (Rosenthal et al., 1987), using $0.02 \%$ digitonin to release mitochondria from the synaptosomal fraction. The whole brain except for the cerebellum was immediately removed, washed and homogenized at $4{ }^{\circ} \mathrm{C}$ in $10 \mathrm{~mL}$ of isolation medium $(225 \mathrm{mM}$ mannitol, $75 \mathrm{mM}$ sucrose, $5 \mathrm{mM}$ Hepes, $1 \mathrm{mM}$ EGTA, $1 \mathrm{mg} / \mathrm{ml}$ defatted BSA, pH 7.4) containing $5 \mathrm{mg}$ of the bacterial protease (Subtilisin A, type VIII from Bacillus licheniformis, Sigma). Single brain homogenates were brought to $30 \mathrm{ml}$ and then centrifuged at $746 \mathrm{~g}$ (Sorvall RC-5B
Refrigerated Superspeed Centrifuge) for $5 \mathrm{~min}$. The pellet was resuspended in $10 \mathrm{ml}$ of the isolation medium containing $0.02 \%$ digitonin and centrifuged at $11,950 \mathrm{~g}$ for $10 \mathrm{~min}$. The pellet was then resuspended in $10 \mathrm{ml}$ of resuspension medium ( $225 \mathrm{mM}$ mannitol, $75 \mathrm{mM}$ sucrose, $5 \mathrm{mM}$ Hepes, $\mathrm{pH} 7.4$ ) and centrifuged at $11,950 \mathrm{xg}$ for $5 \mathrm{~min}$. Finally, the mitochondrial pellet was ressuspended in about $200 \mu$ of resuspension medium. Mitochondrial protein was determined by the Biuret method calibrated with bovine serum albumin (Gornall et al., 1949).

\subsection{Lipid peroxidation}

Lipid peroxidation was evaluated following oxygen consumption using a Clarktype electrode in a glass chamber with magnetic stirring, at $30^{\circ}$. Mitochondria ( $1 \mathrm{mg}$ and $0.8 \mathrm{mg}$ for liver and brain, respectively) were pre-incubated for $3 \mathrm{~min}$ with resveratrol in $1 \mathrm{ml}$ of medium containing $175 \mathrm{mM} \mathrm{KCl}$ and $10 \mathrm{mM}$ Tris-Cl ( $\mathrm{pH}=7.4$ ), supplemented with $2 \mu \mathrm{M}$ rotenone (in the presence or absence of resveratrol) to inhibit mitochondrial respiration induced by endogenous substrates. Membrane lipid peroxidation was initiated by adding $1 \mathrm{mM}$ adenosine diphosphate (ADP)/0.1 mM Fe ${ }^{2+}$ as oxidizing pair. Controls (basal levels) in absence of ADP/Fe $\mathrm{Fe}^{2+}$, were performed under the same conditions. Lipid peroxidation was also evaluated by thiobarbituric acid reactive species (TBARS) generation according to a modified procedure (Santos et al., 2001). Briefly, aliquots of mitochondrial suspension were obtained $10 \mathrm{~min}$ after the addition of $\mathrm{ADP} / \mathrm{Fe}^{2+}$ and added to $0.5 \mathrm{ml}$ of ice-cold $40 \%$ trichloroacetic acid. Then, $2 \mathrm{ml}$ of aqueous thiobarbituric acid $(0.67 \%)$ containing $0.001 \%$ of 2.6 -di-tert-butyl-p-cresol was added to the samples. The mixtures were heated at $90^{\circ}$ for $10 \mathrm{~min}$ and the supernatant fractions were collected and the absorbance read at $530 \mathrm{~nm}$ in a Spectronic 21 spectrophotometer (Bausch \& Lomb, NY, USA). The amount of TBARS formed was calculated using a molar extinction coefficient of $1.56 \times 10^{-5} \mathrm{~mol}^{-1} \mathrm{~cm}^{-1}$ and expressed as nmol TBARS/mg protein (Moreira et al., 2011; Santos et al., 2001).

\subsection{Hydrogen peroxide generation}

Hydrogen peroxide $\left(\mathrm{H}_{2} \mathrm{O}_{2}\right)$ generation was measured fluorimetrically using a modification of a previously described method (Barja, 2002). Briefly, mitochondria were incubated with $1.5 \mathrm{mM}$ of phosphate buffer, $\mathrm{pH} 7.4$, containing $0.1 \mathrm{mM}$ EGTA, $145 \mathrm{mM} \mathrm{KCl}, 30 \mathrm{mM}$ Hepes, and $0.1 \mathrm{mM}$ homovalinic acid and $6 \mathrm{U} / \mathrm{ml}$ horseradish peroxidase. Resveratrol was incubated for 3 mins with mitochondrial fractions. The reactions were initiated by adding $5 \mathrm{mM} / 2.5 \mathrm{mM}$ glutamate/malate. The fluorescence was measured with $312 \mathrm{~nm}$ as excitation wavelength and $420 \mathrm{~nm}$ as emission wavelength in Victor X3 Multilabel reader (Perkin Elmer, Waltham, USA). Hydrogen peroxide generation was calculated using a standard curve of $\mathrm{H}_{2} \mathrm{O}_{2}$ freshly prepared. The standards and the samples were incubated under the same conditions.

\subsection{Mitochondrial respiration}

Oxygen consumption was measured polarographically with a Clark-type Oxygen electrode connected to a recorder in a thermostated water-jacketed closed chamber with magnetic stirring. The reactions were performed at $30^{\circ} \mathrm{C}$ in $1 \mathrm{ml}$ of standard respiratory medium with $1 \mathrm{mg}$ of liver mitochondria or $0.5 \mathrm{mg}$ of brain mitochondria. For liver mitochondria, the reaction medium used was composed by $130 \mathrm{mM}$ sucrose, $50 \mathrm{mM} \mathrm{KCl}, 2.5 \mathrm{mM} \mathrm{KH}_{2} \mathrm{PO}_{4}, 5 \mathrm{mM}$ Hepes and $2 \mathrm{mM} \mathrm{MgCl}$; for brain mitochondria, the reaction medium was composed of $100 \mathrm{mM}$ sucrose, $100 \mathrm{mM} \mathrm{KCl}, 2 \mathrm{mM} \mathrm{KH} \mathrm{PO}_{4}, 5 \mathrm{mM}$ Hepes and $0.01 \mathrm{mM}$ EGTA (pH 7.4). Respiration was initiated with $5 \mathrm{mM}$ glutamate $/ 2.5 \mathrm{mM}$ malate and state 3 started by adding ADP $(150 \mathrm{nmol} / \mathrm{mg}$ protein for brain mitochondria and $125 \mathrm{nmol} / \mathrm{mg}$ protein for liver mitochondria). Respiration rates were obtained assuming an oxygen concentration of $236 \mathrm{nmol} \mathrm{O}_{2} / \mathrm{ml}$ in the experimental medium at $30^{\circ} \mathrm{C}$ (Rasmussen and Rasmussen, 2003). The respiratory state 2 (oxygen consumption before ADP addition - v2), state 3 (oxygen consumption in the presence of ADP - v3), state 4 (oxygen consumption after ADP phosphorylation - v4) and respiratory control ratio $(\mathrm{RCR}=$ state $3 /$ state 4$)$ were obtained according to Chance and Williams (1956). The ADP/O ratio is expressed as the ratio between the amount of ADP added and the oxygen consumed during v3. Resveratrol was pre-incubated with mitochondrial suspension for $3 \mathrm{~min}$ before ADP addition.

In order to determine the possible site of resveratrol interaction, mitochondrial fractions were frozen/thawed three times and oxygen consumption was assessed by using a Clark oxygen type electrode in $1 \mathrm{ml}$ of reaction medium composed by $130 \mathrm{mM}$ sucrose, $50 \mathrm{mM} \mathrm{KCl}, 2.5 \mathrm{mM} \mathrm{KH}_{2} \mathrm{PO}_{4}, 5 \mathrm{mM}$ Hepes and $2 \mathrm{mM} \mathrm{MgCl}$ (liver

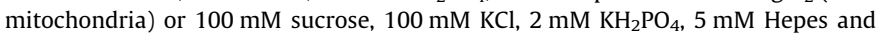
$0.01 \mathrm{mM}$ EGTA (brain mitochondria). One or $0.8 \mathrm{mg}$ mitochondrial protein was used for liver and brain preparations, respectively. Mitochondrial lysates were incubated with resveratrol for three minutes. The direct effects on complex I-sustained respiration were measured in the presence of $1 \mathrm{mM}$ of $\mathrm{NADH}$, while direct effects on complex II-sustained respiration were assessed in the presence of $5 \mathrm{mM}$ of succinate and $2 \mu \mathrm{M}$ rotenone. 


\subsection{Mitochondrial transmembrane electric potential}

The mitochondrial transmembrane electric potential $(\Delta \psi \mathrm{m})$ was estimated by the transmembrane distribution of the lipophilic cation tetraphenylphosphonium $\left(\mathrm{TPP}^{+}\right.$) by using a selective electrode prepared as previously described following the Kamo instructions (Kamo et al., 1979), using an $\mathrm{Ag} / \mathrm{AgCl}_{2}$-saturated electrode as reference. $\mathrm{TPP}^{+}$uptake was measured from the decrease in $\mathrm{TPP}^{+}$concentration in the medium as sensed by the electrode (Oliveira et al., 2004). Mitochondria (1 or $0.5 \mathrm{mg} / \mathrm{ml}$ ) were incubated in the standard reaction medium supplemented with $3 \mu \mathrm{M} \mathrm{TPP}{ }^{+}$and energized by the addition of $5 \mathrm{mM}$ glutamate $/ 2.5 \mathrm{mM}$ malate. After a steady-state distribution of $\mathrm{TPP}^{+}$was reached (after about $1 \mathrm{~min}$ of recording), ADP was added and $\Delta \psi \mathrm{m}$ fluctuations recorded. Resveratrol was pre-incubated with mitochondrial suspension for $3 \mathrm{~min}$ before ADP addition.

\subsection{Complex I activity}

The maximal activity of mitochondrial complex I was assessed in disrupted mitochondrial preparations after three cycles of freezing/thawing. One hundred

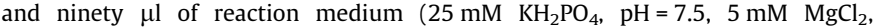
$300 \mu \mathrm{M} \mathrm{KCN}$ ), supplemented with $4 \mu \mathrm{M}$ antimycin $\mathrm{A}, 3 \mathrm{mg} / \mathrm{ml} \mathrm{BSA}, 60 \mu \mathrm{M}$ coenzyme $\mathrm{Q}_{1}, 160 \mu \mathrm{M}$ DCPIP and $10 \mu \mathrm{g} / \mathrm{ml}$ of brain or liver mitochondria were transferred to a 96-well plate. One micromolar of complex I inhibitor rotenone, $25 \mu \mathrm{M}$ resveratrol or $5 \mu \mathrm{l} \mathrm{EtOH}$ (vehicle) were added to the respective wells. Enzymatic activity of brain and liver preparations was measured through a decrease in absorbance of DCPIP after the addition of $100 \mu \mathrm{M}$ fresh-prepared NADH in a Victor X3 plate reader (Perkin Elmer, Waltham, USA) at $600 \mathrm{~nm}$. Enzyme activity was calcu- lated by using the slope achieved during the linear phase (15 cycles). Specific complex I activity was calculated through the difference with the basal activity in the presence of rotenone.

\subsection{Statistical analysis}

The results are representative of at least three different preparations from individual animals as described in the legends of the figures. Values are presented as mean \pm SEM and were compared by two-way ANOVA followed by Bonferroni post-test or t-student test. A value of $p<0.05$ was considered significant.

\section{Results}

\subsection{Resveratrol decreases lipid peroxidation in brain and liver mitochondria}

The effects of resveratrol on oxidative damage were assessed by mitochondria membrane peroxidation induced by the pro-oxidant pair $\mathrm{ADP} / \mathrm{Fe}^{2+}$ (Fig. 1). This effect was evaluated by measuring oxygen consumption (Fig. 1A) and TBARS formation (Fig. 1B). In the absence of resveratrol and after the addition of $\mathrm{ADP} / \mathrm{Fe}^{2+}$, it is possible to observe a two-phase kinetic in oxygen consumption: an initial phase with a slower oxygen consumption that is followed

\section{A}

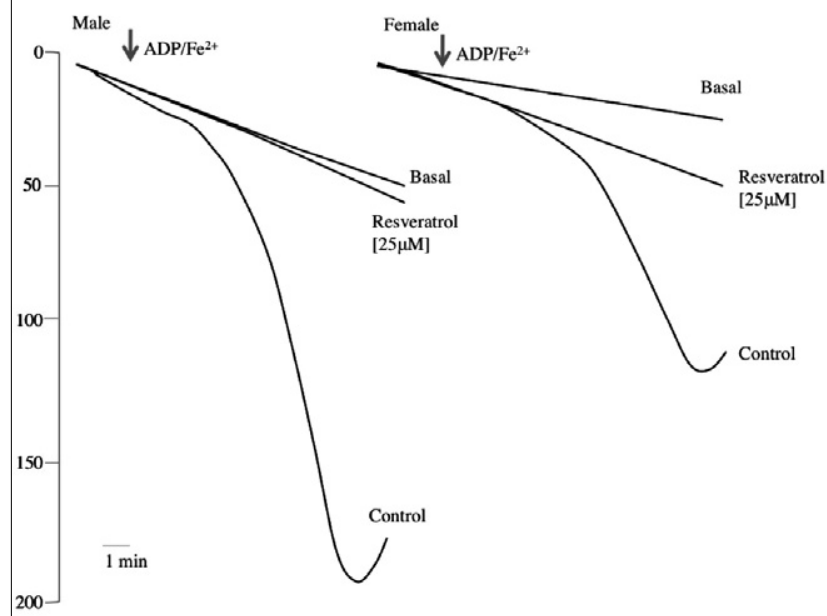

B

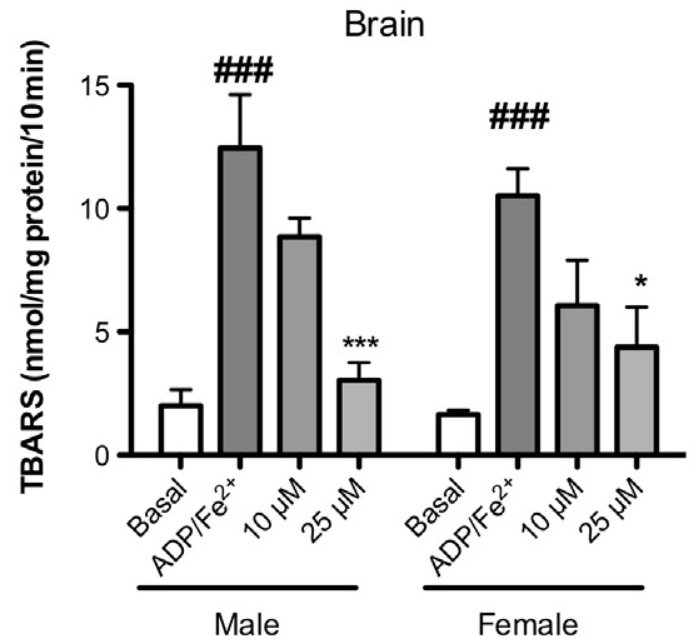

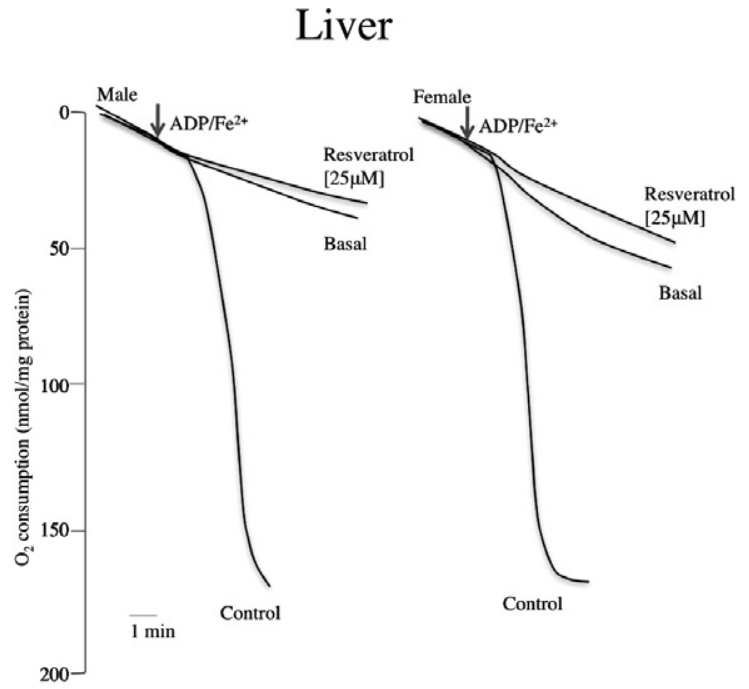

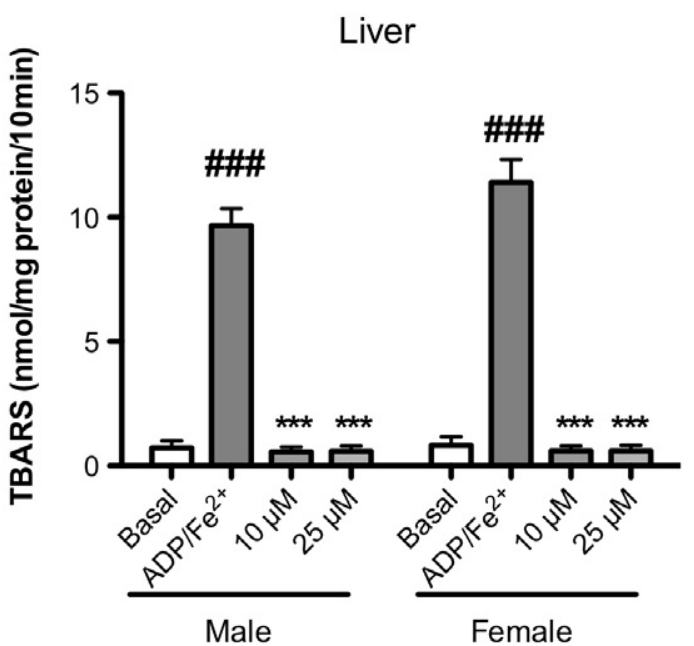

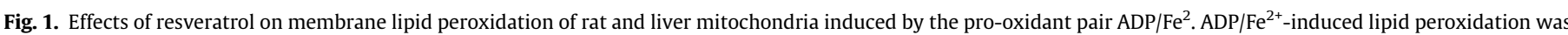

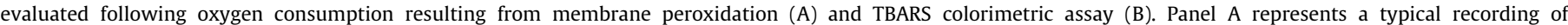


significance: ${ }^{\# \# \#} p<0.001$ compared with the respective basal levels, ${ }^{*} p<0.05,{ }^{* * *} p<0.001$ compared with the mitochondria in the presence of the pair ADP/Fe ${ }^{2+}$. 
A

\section{Brain}



B

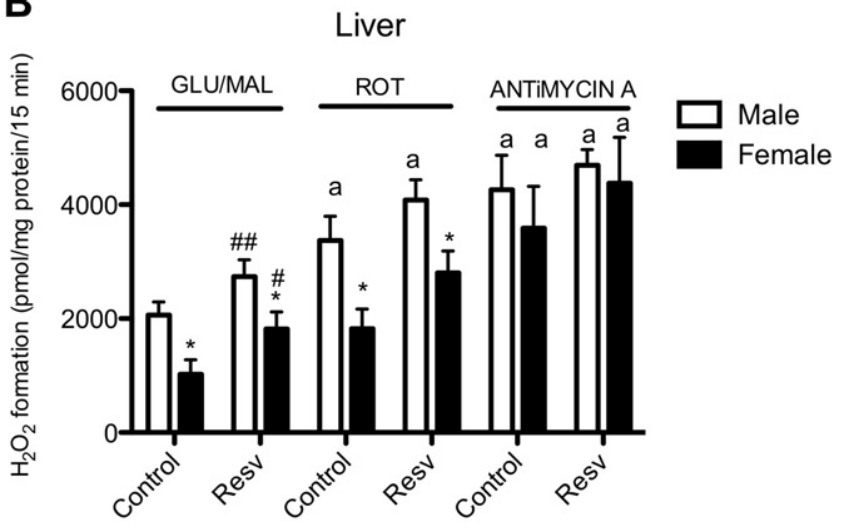

Fig. 2. Effect of resveratrol on mitochondrial hydrogen peroxide $\left(\mathrm{H}_{2} \mathrm{O}_{2}\right)$ production. The production of hydrogen peroxide by the mitochondrial respiratory chain was evaluated as described in the materials and methods section. Mitochondrial fractions from brain $(0.5 \mathrm{mg})$ and liver $(1 \mathrm{mg})$ of male and female Wistar-Han rats were incubated with standard respiratory medium in the presence or absence of resveratrol. Basal levels of $\mathrm{H}_{2} \mathrm{O}_{2}$ production were determined in the absence of mitochondrial substrates. Glutamate/malate $(5 \mathrm{mM} / 2.5 \mathrm{mM})$ was used as substrate. Rotenone $(1.0 \mu \mathrm{M})$ and antimycin $\mathrm{A}(0.5 \mu \mathrm{M})$ were used to increase $\mathrm{H}_{2} \mathrm{O}_{2}$ production. Data represent mean \pm SEM from independent experiments. Statistical significance: ${ }^{*} p<0.05$, when compared with respective gender preparation, ${ }^{*} p<0.05$, ${ }^{\# \#} p<0.01$ when compared with respective control, ${ }^{\text {a }} p<0.05$, aaa $p<0.001$ when compared with the absence of inhibitors.

by a rapid oxygen consumption phase (Fernandes et al., 2006). The initial phase is likely due to the time needed to generate the perferryl ion complex that has been suggested to be the responsible for the initiation of lipid peroxidation. The quickly oxygen consumption is due to the oxidation of the polyunsaturated fatty acid acyl chain of the phospholipidic membrane by ROS, leading to the propagation of lipid peroxidation (Fernandes et al., 2006). As observed in Fig. 1A, in both genders and both organs, the pro-oxidant pair $\mathrm{ADP} / \mathrm{Fe}^{2+}$ induced an increase in oxygen consumption that is decreased in the presence of resveratrol to nearly no-oxidant basal levels. The data are in accordance with the results obtained by measuring TBARS formation. Pre-incubation with the highest concentration of resveratrol $25 \mu \mathrm{M}$ ) lead to a decrease in TBARS production in both organs and genders (brain mitochondria, males: $12.5 \pm 2.5$ vs. $3.0 \pm 0.7$, brain mitochondria females: $10.5 \pm 1.1$ vs. $4.4 \pm 1.6$; male mitochondria, liver: $9.7 \pm 0.7$ vs. $0.6 \pm 0.2$, female mitochondria, liver: $11.4 \pm 0.9$ to $0.6 \pm 0.2 \mathrm{nmol}$ TBARS/mg protein, without vs. with resveratrol) (Fig. 1B). For the lowest concentration used $(10 \mu \mathrm{M})$, resveratrol had no effect on brain mitochondrial fractions, as opposed to liver mitochondria, where protection against lipid peroxidation-induced TBARS generation was observed for both genders.
3.2. Resveratrol increases the mitochondrial $\mathrm{H}_{2} \mathrm{O}_{2}$ production in liver

Liver and brain mitochondria from male and female rats were incubated with resveratrol in order to investigate the effects of the compound on hydrogen peroxide generation by the respiratory chain.

In both genders, treatment of mitochondria with rotenone (a complex I inhibitor) or antimycin A (a complex III inhibitor) maximally induces $\mathrm{H}_{2} \mathrm{O}_{2}$ generation, as expected (Chen et al., 2003; Leloup et al., 2006) (Fig. 2). When analyzing $\mathrm{H}_{2} \mathrm{O}_{2}$ production in untreated mitochondria (Fig. 2), liver mitochondria from female rats have generally a lower basal $\mathrm{H}_{2} \mathrm{O}_{2}$ generation when compared with preparations from male rats. The same effect was not observed in brain mitochondrial fractions. When resveratrol was added to the different experimental groups, some interesting effects were observed. When added to liver mitochondria, resveratrol increased $\mathrm{H}_{2} \mathrm{O}_{2}$ generation when glutamate-malate was used alone (Fig. 2B), in both preparations from male and female rats. In brain mitochondrial fractions (male only), resveratrol amplified the effect of antimycin $\mathrm{A}$ in increased $\mathrm{H}_{2} \mathrm{O}_{2}$ generation (Fig. $2 \mathrm{~A}$ ).

We also compared directly liver and brain mitochondrial fractions from both genders in terms of basal activity of glutathione peroxidase (GPx) and glutathione content. We have measured that in general, liver mitochondrial have increased GPx, with fractions from females having the highest activity $(345.80 \pm 46.01$ to $216.40 \pm 17.71 \mathrm{U} / \mathrm{mg}$ protein of male fractions, Fig. S1). No differences were observed in terms of GSH, GSSG or GSH/GSSG ratio (Table S1).

\subsection{Resveratrol decreases state 3 respiration in brain mitochondria}

The next question in this work led us to investigate direct effects of resveratrol on liver and brain mitochondrial respiration for both genders. In the absence of resveratrol, no differences between genders were observed. The addition of resveratrol $(10,25 \mu \mathrm{M})$ to freshly isolated brain mitochondria induced consumption decrease of state 3 respiration from $44.0 \pm 1.8$ to $33.5 \pm 3.5 \mathrm{nmol} \mathrm{O}_{2} / \mathrm{mg}$ protein for males and from $44.1 \pm 3.0$ to $32.8 \pm 3.7 \mathrm{nmol} \mathrm{O}_{2} / \mathrm{mg}$ protein for preparations from female animals (Fig 3A). The alterations in respiration state 3 in brain mitochondria were not reflected in the RCR (Fig. 3C), may be due to the fact that the oxygen consumption has also has a tendency to be decreased by resveratrol during state 4 ( $p=0.0591$, Fig. 3B). No alterations were observed for liver mitochondria (Fig. 3 ). The $\mathrm{ADP} / \mathrm{O}$, a measure of the efficiency of oxidative phosphorylation, was not altered after resveratrol incubation for any of the groups studied (Fig. 3D).

\subsection{Mitochondrial membrane potential during ADP phosphorylation is affected by resveratrol}

Gender did not influence the different end-points regarding mitochondrial transmembrane electric potential (Fig. 4). Resveratrol did not affect the maximum transmembrane electric potential developed by mitochondria for both genders and organs (Fig. 4A). However, the depolarization induced by ADP is decreased by resveratrol at $25 \mu \mathrm{M}$ in brain and liver mitochondria (Fig. 4B). In brain mitochondria, the decrease was from $15.3 \pm 1.0$ to $10.3 \pm 2.7$ (preparations from males) and $14.2 \pm 1.0$ to $9.3 \pm 1.5$ (preparations from females), numbers in $(-\mathrm{mV})$. When using liver mitochondria, the decrease was from $20.4 \pm 2.1$ to $15.7 \pm 0.8$ (preparations from male) and from $19.5 \pm 0.4$ to $16.2 \pm 0.4$ (preparations from females), results in $(-\mathrm{mV})$. Although there is not a statistical difference, the lag phase showed a tendency to be increased in brain mitochondria from male rats when incubated with $25 \mu \mathrm{M}$ resveratrol $(p=0.0591)$. 

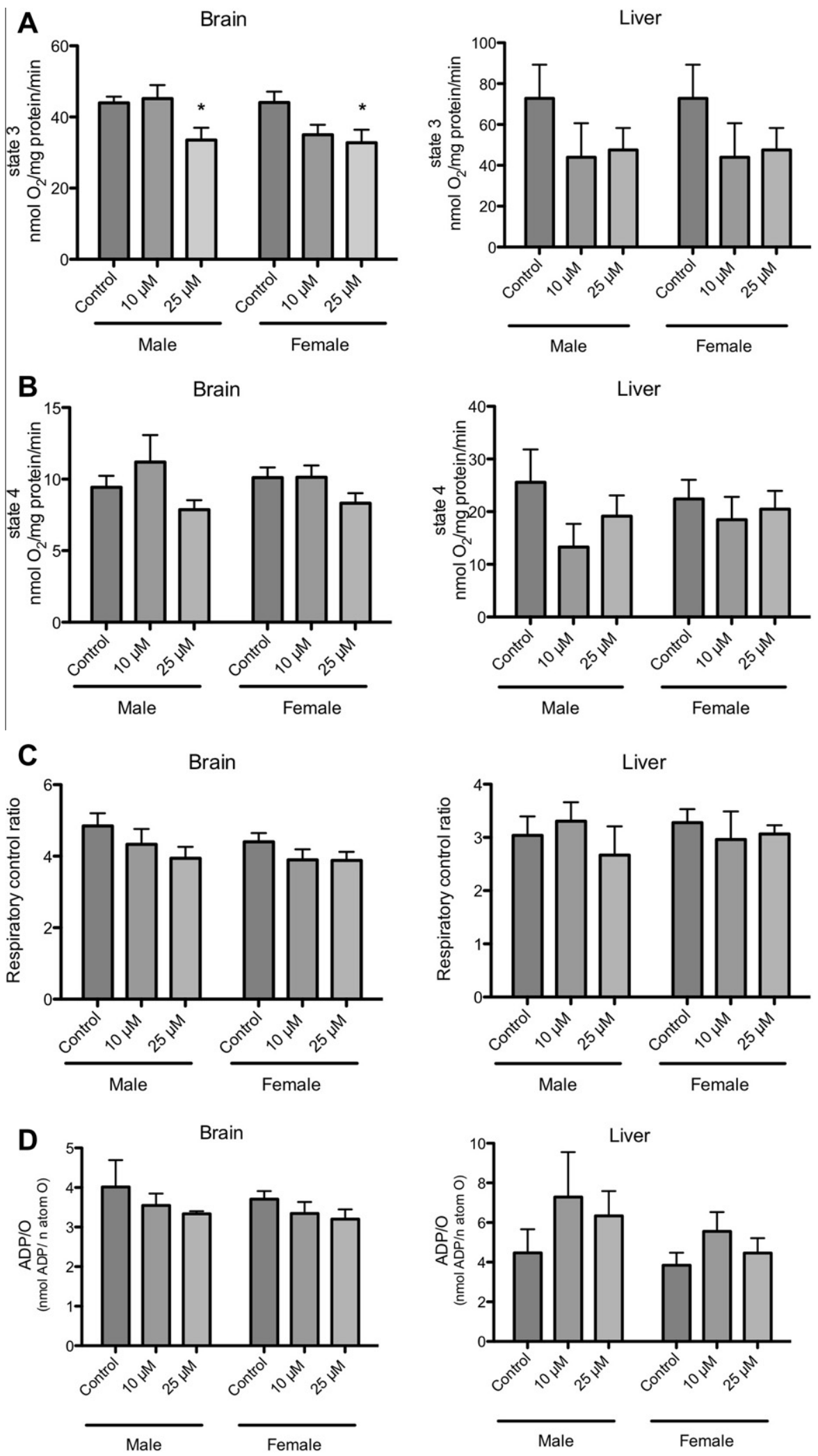

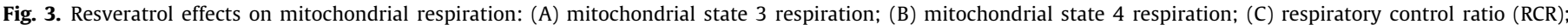

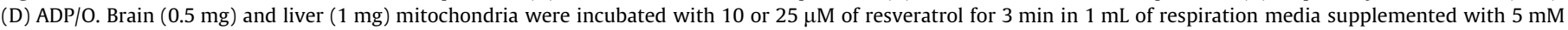

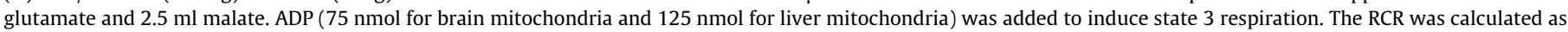

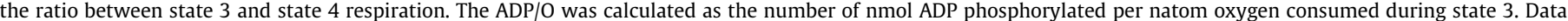
represent mean \pm SEM from four to six independent experiments. Statistical significance: ${ }^{*} p<0.05$, when compared with the respective control. 

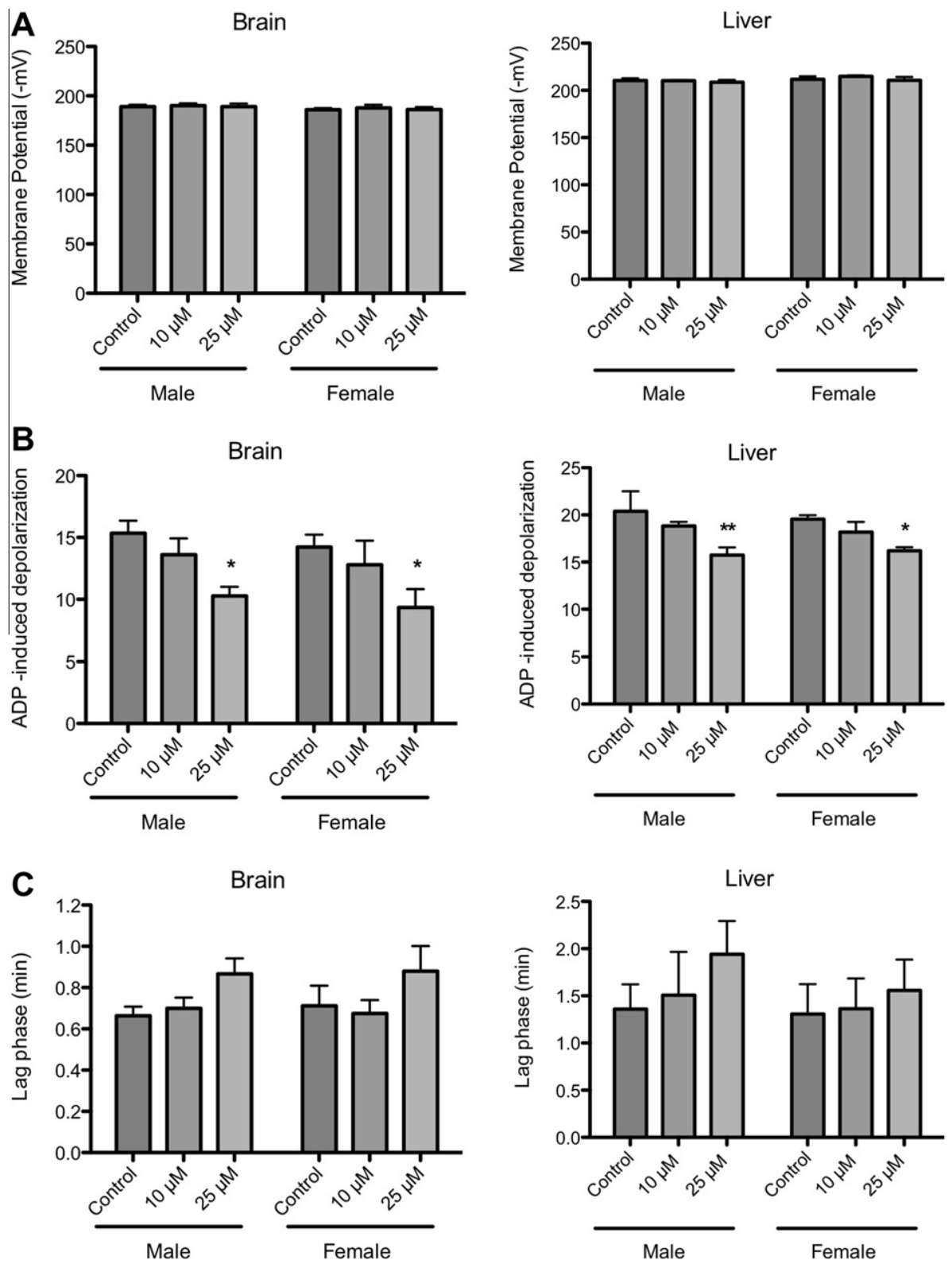

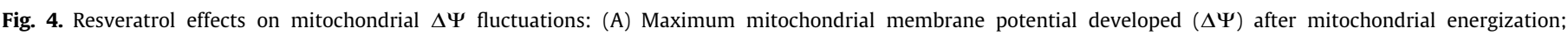

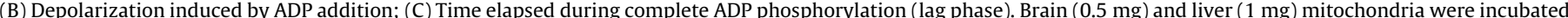

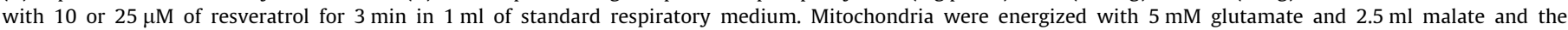


independent experiments. Statistical significance ${ }^{*} p<0.05$ and ${ }^{* *} p<0.01$ when compared with the respective control.

\subsection{Resveratrol demonstrates a direct effect on mitochondrial complex I}

By using disrupted mitochondrial membranes and specific substrates, one can evaluate distinct sites of drug-induced toxicity. Resveratrol decreases oxygen consumption in frozen/thawed mitochondrial preparations from liver and brain when using NADH as substrate (Fig 5A and E). Preparations from brains of male rats showed a resveratrol-induced decrease in oxygen consumption from $56.3 \pm 4.4$ to $48.81 \pm 3.6 \mathrm{nmol} \mathrm{O}_{2} / \mathrm{mg}$ protein/ min; while a decrease from $44.8 \pm 5.2$ to $36.2 \pm 3.4 \mathrm{nmol} \mathrm{O} / \mathrm{mg}$ protein/ min was observed in brain preparations from female rats. When investigating liver mitochondrial preparations, resveratrol also inhibited complex I-sustained respiration in male $(24.7 \pm 1.7$ to $21.0 \pm$ $1.4 \mathrm{nmol} \mathrm{O}$ consumed $/ \mathrm{mg} / \mathrm{min})$ and female rats $(19.9 \pm 0.9$ to $16.4 \pm 0.9 \mathrm{nmol} \mathrm{O}_{2}$ consumed $\left./ \mathrm{mg} / \mathrm{min}\right)$. Interestingly, NADH- sustained oxygen consumption in both tissues was higher in preparations from males when compared with preparations from female animals. The same experiments were performed by using the complex II substrate succinate (in the presence of complex I inhibitor rotenone). In this case, resveratrol had no effect on succinate-induced mitochondrial respiration.

In parallel, the maximum activity of complex I was investigated using a colorimetric method. For both organs and genders, resveratrol decreased complex I specific activity. In brain mitochondria, a decrease of $22 \%$ and $11 \%$ was observed in preparations from male and female preparations, respectively. A higher magnitude effect was observed in liver mitochondria, where the activity in the presence of resveratrol decreased $73 \%$ for male preparations and practically $100 \%$ in the case of preparations from female rats. The maximal activity of complex I did not differ between genders. 
A

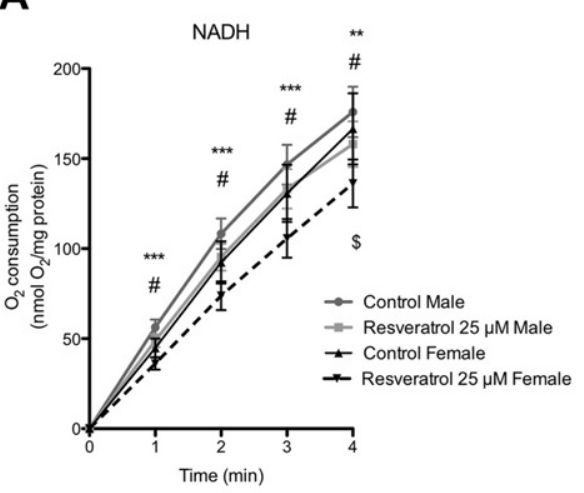

B

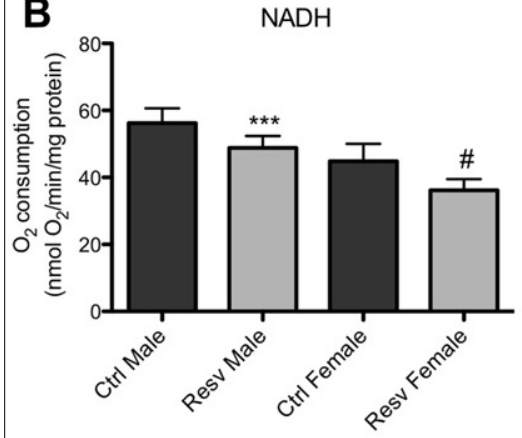

C Succinate (+Rotenone)

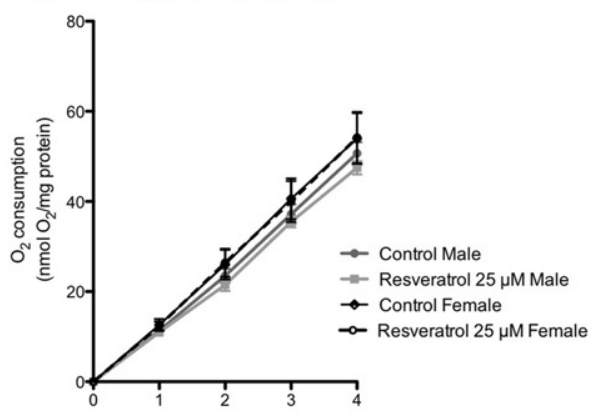

D

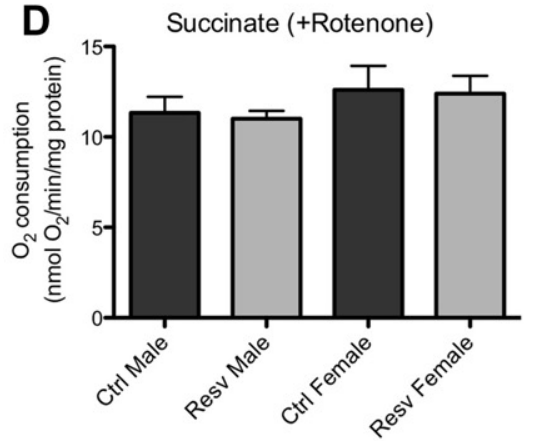

E Liver

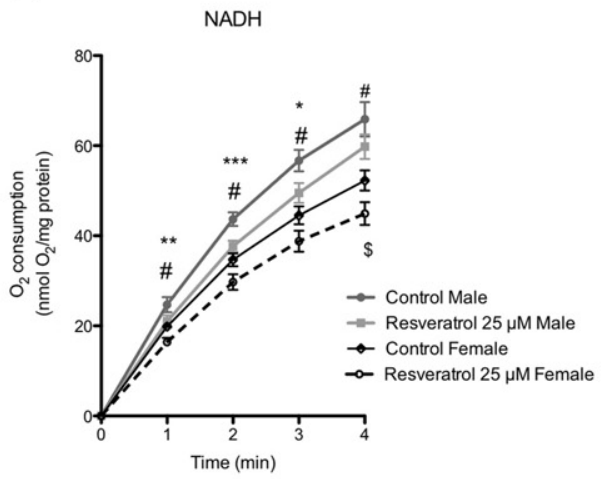

F NADH

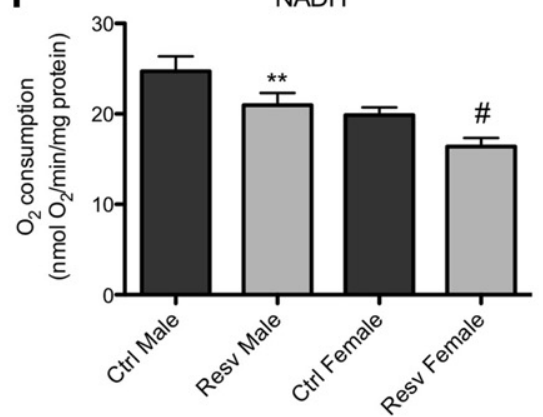

G

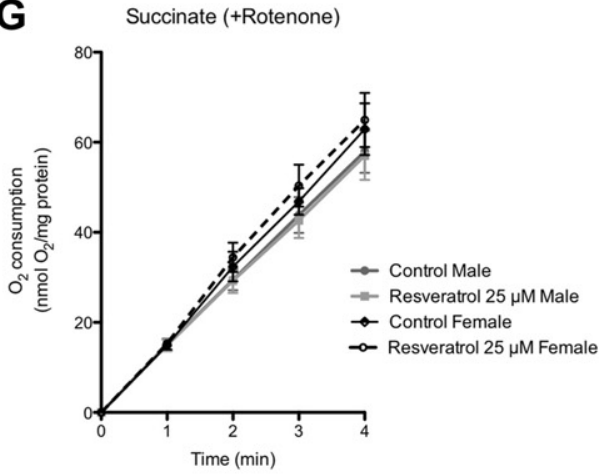

H

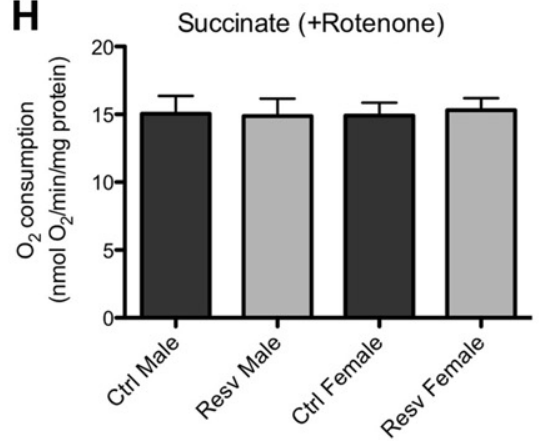

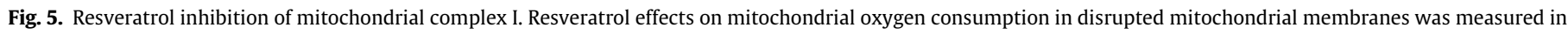

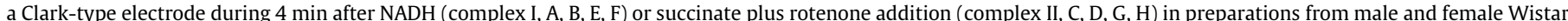

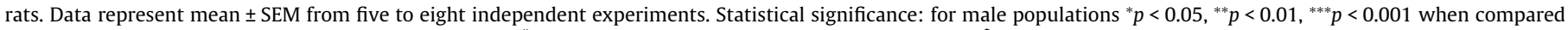
with the respective control and for female preparations ${ }^{\#} p<0.05$ when compared with respective control, ${ }^{\$} p<0.05$, compared with male. 


\section{Discussion}

There is a growing amount of studies focusing on natural compounds and their possible benefits in terms of human health. Although the antioxidant, anti-inflammatory and anti-tumoral effects of resveratrol (Delmas et al., 2006; Pervaiz and Holme, 2009) have been widely described, the cellular toxicity was not yet completely evaluated, especially regarding mitochondrial bioenergetics.

Following previous works showing protective effects of resveratrol on brain and liver (Morin et al., 2003; Park et al., 2010; Pervaiz and Holme, 2009), the present work investigates its toxicity on isolated brain and liver mitochondria at concentrations that act to decrease oxidative stress. The use of brain and liver mitochondria was due to the fact that resveratrol is able to cross the BBB (Wang et al., 2002) and it is highly metabolized in the liver (Walle, 2011), respectively. The present work highlights the relevance of using mitochondrial-isolated fractions from male and female rats, which is very rare in the literature. Although we did not observe basal differences in mitochondrial preparations from both genders in most of the parameters investigated, except when measuring maximal respiration due to freezing/thawing cycles, it is known that estrogens have protective effects, enhancing antioxidant defenses and decreasing mitochondrial dysfunction (Borras et al., 2007; Eskes and Haanen, 2007; Vina et al., 2011). For instance, in liver mitochondrial the $\mathrm{H}_{2} \mathrm{O}_{2}$ levels are lower in females than in males (Fig. 2). This can be due to the activity of GPx, which is augmented in liver preparations from females (Fig. S1), although no differences were measured in brain or in reduced and oxidized GSH (Table S1). Female rats have better mitochondrial capacity showing less oxidative stress generation based in estrogen levels (Borras et al., 2003; Guevara et al., 2011). Sex hormones have neuroprotective effects, which have also been shown to increase mitochondrial efficiency (Nilsen and Brinton, 2004). Besides, the differences in gender, we aimed to discuss the different effects of resveratrol and to observe its effects on mitochondrial function for concentrations known to act as antioxidant. Our results confirm previous studies (Aftab et al., 2010; Ghanim et al., 2011; Kelsey et al., 2010; Toklu et al., 2010) regarding the antioxidant proprieties of resveratrol (Fig. 1). Our data shows that resveratrol indeed acts as an antioxidant by the measurement of TBARS and by following oxygen consumption during lipid peroxidation. In both cases (Fig. 1), resveratrol was able to decrease lipid peroxidation, disregarding the gender. Resveratrol increases $\mathrm{H}_{2} \mathrm{O}_{2}$ generation in both male and female liver mitochondria in the presence of the substrate (glutamate-malate) alone. The effect is not observed with brain mitochondria (Fig. 2), except when mitochondria are incubated with complex III inhibitor, antimycin A (Fig. 2A). The resveratrol effects on liver mitochondria in terms of $\mathrm{H}_{2} \mathrm{O}_{2}$ generation can be explained by a direct action on complex I, or instead, as previously reported, by modulation of mitochondrial Manganesesuperoxide dismutase (Robb et al., 2008), increasing the flux of hydrogen peroxide production.

Concerning effects of resveratrol on mitochondrial bioenergetics, Zini et al., showed that resveratrol inhibits brain mitochondrial respiratory chain at the complexes I-III span (Zini et al., 1999). In this work, the enzymatic activity of ubiquinol cytochrome $c$ reductase in the presence of resveratrol was decreased by $20 \%$ (Zini et al. 1999). The authors suggest that resveratrol can preserve mitochondrial functions by three different mechanisms: antioxidantmediated effect, complex III direct effect and membrane stabilizing effects (Zini et al., 2002). A decrease of state 3 in brain mitochondria of female and male rats was observed in the presence of resveratrol (Fig. 3). Since the maximal transmembrane electric potential developed was not affected by resveratrol (Fig. 4), this
Table 1

Effect of resveratrol on brain and liver mitochondrial respiratory complex I maximum activity. Activity expressed as nmol DCPIP/min/mg protein.

\begin{tabular}{llllll}
\hline & Male & & & Female & \\
\cline { 2 - 3 } \cline { 5 - 6 } & Ctrl & Resv & & Ctrl & Resv \\
\hline Brain & $135.7 \pm 16.82$ & $106.6 \pm 13.05^{*}$ & & $112.4 \pm 4.724$ & $99.61 \pm 4.3^{*}$ \\
Liver & $49.74 \pm 12.14$ & $13.65 \pm 13.27^{*}$ & $52.12 \pm 4.97$ & $0.00 \pm 12.47^{*}$ \\
\hline
\end{tabular}

Data are the mean \pm SEM of four independent experiment. Statistical significance: ${ }^{*} p<0.05$ when compared with the respective control.

suggests that the respiratory chain was not largely inhibited, although we cannot exclude that increased $\mathrm{H}_{2} \mathrm{O}_{2}$ generation results from inhibition of complex I and/or III. Instead, the results from both respiration (Fig. 3) and transmembrane electric potential (Fig. 4) suggest an effect on the phosphorylative system, including in the adenine nucleotide transporter, the phosphate transporter, or even with the ATP synthase (Gledhill and Walker, 2005). Interestingly, inhibition of state 3 respiration was not observed in liver mitochondria. Although the reason for this difference is still unclear, it may have to do with differential accumulation of resveratrol in mitochondrial membranes from both gender groups or with the different number of target complex subunits present in both preparations.

We further described that resveratrol inhibits mitochondrial respiration induced by NADH, but not succinate, in freeze/thawed preparations, which indirectly allows to study the maximal respiration rate (Fig. 5). In accordance, resveratrol also reduced complex I specific activity as followed by the reduction of DCPIP (Table 1). This data demonstrates that resveratrol acts as a complex I inhibitor in mitochondrial preparations from both organs and genders. Interestingly, although resveratrol induced a larger inhibition of complex I activity in liver freeze/thawed preparations, the same type of effect was not visible when using intact mitochondria (Fig. 3). This may imply that the site of resveratrol inhibition on complex I faces the matrix side, being more accessible when mitochondrial membranes are disrupted to perform specific assays with NADH. The data also supplies evidence that resveratrol may also target complex I besides the already described complex III (Zini et al., 1999), thus contributing to increase the generation of hydrogen peroxide by the respiratory chain (Fig. 2 ).

The results may also suggest that increased generation of $\mathrm{H}_{2} \mathrm{O}_{2}$ in liver and, under some conditions, in brain mitochondria by resveratrol can act to stimulate several signaling pathways, including those related with antioxidant defenses. The effects of resveratrol in the metabolism of different organs and gender-mediated effects should be explored in further detail to determine potential toxic effects and mechanisms by which resveratrol is described to cell fitness.

\section{Conflict of Interest}

The authors declare that there are no conflicts of interest.

\section{Acknowledgements}

This work is supported by PTDC/AGR-ALI/108326/2008 to M.S.S. from the Portuguese Foundation for Science and Technology, FEDER/Compete/National Funds. A.C.M., A.M.S. and V.A.S. are recipient of SFRH/BD/33892/2009, SFRH/BD/76086/2011 and SFRH/BPD/ $31549 / 2006$ fellowships, respectively. The authors acknowledge Dr. Paulo J. Oliveira for reviewing the manuscript and Gonçalo C. Pereira and and Dr. António J. Moreno for the useful discussions. 


\section{Appendix A. Supplementary data}

Supplementary data associated with this article can be found, in the online version, at http://dx.doi.org/10.1016/j.fct.2012.11.031.

\section{References}

Aftab, N., Likhitwitayawuid, K., Vieira, A., 2010. Comparative antioxidant activities and synergism of resveratrol and oxyresveratrol. Nat. Prod. Res. 24, 1726-1733.

Agarwal, B., Baur, J.A., 2011. Resveratrol and life extension. Ann. N. Y. Acad. Sci. $1215,138-143$.

Annabi, B., Lord-Dufour, S., Vezina, A., Beliveau, R., 2012. Resveratrol targeting of carcinogen-induced brain endothelial cell inflammation biomarkers MMP-9 and COX-2 is Sirt1-independent. Drug Target Insights 6,1-11.

Barja, G., 2002. The quantitative measurement of $\mathrm{H}_{2} \mathrm{O}_{2}$ generation in isolated mitochondria. J. Bioenerg. Biomembr. 34, 227-233.

Baur, J.A., Sinclair, D.A., 2006. Therapeutic potential of resveratrol: the in vivo evidence. Nat. Rev. Drug Discov. 5, 493-506.

Borras, C., Gambini, J., Vina, J., 2007. Mitochondrial oxidant generation is involved in determining why females live longer than males. Front Biosci. 12, 1008-1013.

Borras, C., Sastre, J., Garcia-Sala, D., Lloret, A., Pallardo, F.V., Vina, J., 2003. Mitochondria from females exhibit higher antioxidant gene expression and lower oxidative damage than males. Free Radic. Biol. Med. 34, 546-552.

Chan, C.C., Cheng, L.Y., Lin, C.L., Huang, Y.H., Lin, H.C., Lee, F.Y., 2011. The protective role of natural phytoalexin resveratrol on inflammation, fibrosis and regeneration in cholestatic liver injury. Mol. Nutr. Food Res. 55, 1841-1849.

Chance, B., Williams, G.R., 1956. Respiratory enzymes in oxidative phosphorylation. VI. The effects of adenosine diphosphate on azide-treated mitochondria. J. Biol. Chem. 221, 477-489.

Chen, Q., Vazquez, E.J., Moghaddas, S., Hoppel, C.L., Lesnefsky, E.J., 2003. Production of reactive oxygen species by mitochondria: central role of complex III. J. Biol. Chem. 278, 36027-36031.

Dal-Pan, A., Pifferi, F., Marchal, J., Picq, J.L., Aujard, F., 2011. Cognitive performances are selectively enhanced during chronic caloric restriction or resveratrol supplementation in a primate. PLoS ONE 6, e16581.

Delmas, D., Lancon, A., Colin, D., Jannin, B., Latruffe, N., 2006. Resveratrol as a chemopreventive agent: a promising molecule for fighting cancer. Curr. Drug Targets $7,423-442$.

Eskes, T., Haanen, C., 2007. Why do women live longer than men? Eur. J. Obstet. Gynecol. Reprod. Biol. 133, 126-133.

Fernandes, M.A., Custodio, J.B., Santos, M.S., Moreno, A.J., Vicente, J.A., 2006 Tetrandrine concentrations not affecting oxidative phosphorylation protect rat liver mitochondria from oxidative stress. Mitochondrion 6, 176-185.

Fremont, L., 2000. Biological effects of resveratrol. Life Sci. 66, 663-673.

Fukui, M., Choi, H.J., Zhu, B.T., 2010. Mechanism for the protective effect of resveratrol against oxidative stress-induced neuronal death. Free Radic. Biol. Med. 49, 800-813.

Ghanim, H., Sia, C.L., Korzeniewski, K., Lohano, T., Abuaysheh, S., Marumganti, A. Chaudhuri, A., Dandona, P., 2011. A resveratrol and polyphenol preparation suppresses oxidative and inflammatory stress response to a high-fat, highcarbohydrate meal. J. Clin. Endocrinol. Metab.

Gledhill, J.R., Walker, J.E., 2005. Inhibition sites in F1-ATPase from bovine heart mitochondria. Biochem. J. 386, 591-598.

Gornall, A.G., Bardawill, C.J., David, M.M., 1949. Determination of serum proteins by means of the biuret reaction. J. Biol. Chem. 177, 751-766.

Gresele, P., Cerletti, C., Guglielmini, G., Pignatelli, P., de Gaetano, G., Violi, F., 2011. Effects of resveratrol and other wine polyphenols on vascular function: an update. J. Nutr. Biochem. 22, 201-211.

Guevara, R., Gianotti, M., Roca, P., Oliver, J., 2011. Age and sex-related changes in rat brain mitochondrial function. Cell. Physiol. Biochem. 27, 201-206.

Gutierrez-Perez, A., Cortes-Rojo, C., Noriega-Cisneros, R., Calderon-Cortes, E., Manzo-Avalos, S., Clemente-Guerrero, M., Godinez-Hernandez, D., Boldogh, I., Saavedra-Molina, A., 2011. Protective effects of resveratrol on calcium-induced oxidative stress in rat heart mitochondria. J. Bioenerg. Biomembr. 43, 101-107.

He, X., Wang, L., Szklarz, G., Bi, Y., Ma, Q., 2012. Resveratrol inhibits paraquatinduced oxidative stress and fibrogenic response by activating the Nrf2 pathway. J. Pharmacol. Exp. Ther..

Huber, K., Superti-Furga, G., 2011. After the grape rush: sirtuins as epigenetic drug targets in neurodegenerative disorders. Bioorg. Med. Chem.

Kamo, N., Muratsugu, M., Hongoh, R., Kobatake, Y., 1979. Membrane potential of mitochondria measured with an electrode sensitive to tetraphenyl phosphonium and relationship between proton electrochemical potential and phosphorylation potential in steady state. J. Membr. Biol. 49, 105-121.

Kelsey, N.A., Wilkins, H.M., Linseman, D.A., 2010. Nutraceutical antioxidants as novel neuroprotective agents. Molecules $15,7792-7814$

Leloup, C., Magnan, C., Benani, A., Bonnet, E., Alquier, T., Offer, G., Carriere, A. Periquet, A., Fernandez, Y., Ktorza, A., Casteilla, L., Penicaud, L., 2006. Mitochondrial reactive oxygen species are required for hypothalamic glucose sensing. Diabetes 55, 2084-2090.

Moreira, P.I., Custodio, J.B., Nunes, E., Oliveira, P.J., Moreno, A., Seica, R., Oliveira, C.R. Santos, M.S., 2011. Mitochondria from distinct tissues are differently affected by 17beta-estradiol and tamoxifen. J. Steroid Biochem. Mol. Biol. 123, 8-16.

Moreno, A.J., Oliveira, P.J., Nova, C.D., Alvaro, A.R., Moreira, R.A., Santos, S.M., Macedo, T., 2007. Unaltered hepatic oxidative phosphorylation and mitochondrial permeability transition in wistar rats treated with nimesulide: relevance for nimesulide toxicity characterization. J. Biochem. Mol. Toxicol. 21, 53-61.

Morin, C., Zini, R., Albengres, E., Bertelli, A.A., Bertelli, A., Tillement, J.P., 2003. Evidence for resveratrol-induced preservation of brain mitochondria functions after hypoxia-reoxygenation. Drugs Exp. Clin. Res. 29, 227-233.

Nilsen, J., Brinton, R.D., 2004. Mitochondria as therapeutic targets of estrogen action in the central nervous system. Curr. Drug Targets CNS Neurol. Disord. 3, 297313.

Nonomura, S., Kanagawa, H., Makimoto, A., 1963. Chemical constituents of polygonaceous plants. I. Studies on the components of Ko-J O-Kon. (Polygonum Cuspidatum Sieb. Et Zucc.). Yakugaku Zasshi 83, 988-990.

Oliveira, P.J., Esteves, T.C., Seica, R., Moreno, A.J., Santos, M.S., 2004. Calciumdependent mitochondrial permeability transition is augmented in the kidney of Goto-Kakizaki diabetic rat. Diabetes Metab. Res. Rev. 20, 131-136.

Park, J.M., Kim, T.H., Bae, J.S., Kim, M.Y., Kim, K.S., Ahn, Y.H., 2010. Role of resveratro in FOXO1-mediated gluconeogenic gene expression in the liver. Biochem. Biophys. Res. Commun. 403, 329-334.

Pereira, C.V., Moreira, A.C., Pereira, S.P., Machado, N.G. Carvalho, F.S., Sardao, V.A Oliveira, P.J., 2009a. Investigating drug-induced mitochondrial toxicity: biosensor to increase drug safety? Curr. Drug Saf. 4, 34-54.

Pereira, S.P., Pereira, G.C., Moreno, A.J., Oliveira, P.J., 2009b. Can drug safety be predicted and animal experiments reduced by using isolated mitochondrial fractions? Altern. Lab. Anim. 37, 355-365.

Pervaiz, S., 2003. Resveratrol: from grapevines to mammalian biology. FASEB J. 17, 1975-1985.

Pervaiz, S., Holme, A.L., 2009. Resveratrol: its biologic targets and functional activity. Antioxid. Redox Signal. 11, 2851-2897.

Price, N.L., Gomes, A.P., Ling, A.J., Duarte, F.V., Martin-Montalvo, A., North, B.J., Agarwal, B., Ye, L., Ramadori, G., Teodoro, J.S., Hubbard, B.P., Varela, A.T. Davis, J.G., Varamini, B., Hafner, A., Moaddel, R., Rolo, A.P., Coppari, R., Palmeira, C.M., de Cabo, R., Baur, J.A., Sinclair, D.A., 2012. SIRT1 is required for AMPK activation and the beneficial effects of resveratrol on mitochondrial function. Cell Metab. $15,675-690$.

Puente-Maestu, L., Tejedor, A., Lazaro, A., de Miguel, J., Alvarez-Sala, L., GonzalezAragoneses, F., Simon, C., Agusti, A., 2012. Site of mitochondrial ROS production in skeletal muscle of COPD and its relationship with exercise oxidative stress. Am J Respir Cell Mol Biol.

Rasmussen, H.N., Rasmussen, U.F., 2003. Oxygen solubilities of media used in electrochemical respiration measurements. Anal. Biochem. 319, 105-113.

Reale, M., Pesce, M., Priyadarshini, M., Kamal, M.A., Patruno, A., 2012. Mitochondria as an easy target to oxidative stress events in Parkinson's disease. Curr. Drug Targets CNS Neurol. Disord.

Richard, J.L., 1987. Coronary risk factors. The French paradox. Arch Mal Coeur Vaiss 80 Spec No., 17-21.

Rigoulet, M., Yoboue, E.D., Devin, A., 2011. Mitochondrial ROS generation and its regulation: mechanisms involved in $\mathrm{H}(2) \mathrm{O}(2)$ signaling. Antioxid. Redox Signal. 14, 459-468.

Robb, E.L., Page, M.M., Wiens, B.E., Stuart, J.A., 2008. Molecular mechanisms of oxidative stress resistance induced by resveratrol: Specific and progressive induction of MnSOD. Biochem. Biophys. Res. Commun. 367, 406-412.

Rosenthal, R.E., Hamud, F., Fiskum, G., Varghese, P.J., Sharpe, S., 1987. Cerebral ischemia and reperfusion: prevention of brain mitochondrial injury by lidoflazine. J. Cereb. Blood Flow Metab. 7, 752-758.

Santos, M.S., Santos, D.L., Palmeira, C.M., Seica, R., Moreno, A.J., Oliveira, C.R., 2001 Brain and liver mitochondria isolated from diabetic Goto-Kakizaki rats show different susceptibility to induced oxidative stress. Diabetes Metab. Res. Rev. 17, 223-230.

Sebai, H., Sani, M., Aouani, E., Ghanem-Boughanmi, N., 2011. Cardioprotective effect of resveratrol on lipopolysaccharide-induced oxidative stress in rat. Drug Chem. Toxicol. 34, 146-150.

Toklu, H.Z., Sehirli, O., Ersahin, M., Suleymanoglu, S., Yiginer, O., Emekli-Alturfan, E. Yarat, A., Yegen, B.C., Sener, G., 2010. Resveratrol improves cardiovascular function and reduces oxidative organ damage in the renal, cardiovascular and cerebral tissues of two-kidney, one-clip hypertensive rats. J. Pharm. Pharmacol. 62, 1784-1793

Valdecantos, M.P., Perez-Matute, P., Quintero, P., Martinez, J.A, 2010. Vitamin C resveratrol and lipoic acid actions on isolated rat liver mitochondria: all antioxidants but different. Redox Rep. 15, 207-216.

Vina, J., Gambini, J., Lopez-Grueso, R., Abdelaziz, K.M., Jove, M., Borras, C., 2011. Females live longer than males: role of oxidative stress. Curr. Pharm. Des. 17, 3959-3965.

Walle, T., 2011. Bioavailability of resveratrol. Ann. N. Y. Acad. Sci. 1215, 9-15.

Wang, Q., Xu, J., Rottinghaus, G.E., Simonyi, A., Lubahn, D., Sun, G.Y., Sun, A.Y., 2002. Resveratrol protects against global cerebral ischemic injury in gerbils. Brain Res. 958, 439-447.

Xu, X., Chen, K., Kobayashi, S., Timm, D., Liang, Q., 2012. Resveratrol attenuates doxorubicin-induced cardiomyocyte death via inhibition of p70 S6 kinase 1mediated autophagy. J. Pharmacol. Exp. Ther. 341, 183-195.

Zini, R., Morin, C., Bertelli, A., Bertelli, A.A., Tillement, J.P., 1999. Effects of resveratrol on the rat brain respiratory chain. Drugs Exp. Clin. Res. 25, 87-97.

Zini, R., Morin, C., Bertelli, A., Bertelli, A.A., Tillement, J.P., 2002. Resveratrol-induced limitation of dysfunction of mitochondria isolated from rat brain in an anoxiareoxygenation model. Life Sci. 71, 3091-3108. 